\title{
Peripheral levels of circulating progenitor cells correlate with area at risk and microvascular obstruction at cardiac magnetic resonance imaging in patients with ST-elevation myocardial infarction
}

\author{
Italo Porto ${ }^{*}$, Christian Hamilton Craig, Giovanni Luigi De Maria, Antonio Maria Leone, Alessandra Tritarelli, \\ Francesco Burzotta, Claudia Camaioni, Luigi Natale, Luigi Marzio Biasucci, Filippo Crea
}

From 2011 SCMR/Euro CMR Joint Scientific Sessions

Nice, France. 3-6 February 2011

\section{Background}

Circulating progenitor cells (CPC) are bone marrowderived elements with reparative properties known to be mobilised in the context of acute coronary syndromes. Controversy persists over the determinants of their release in the acute phase of ST-elevation myocardial infarction (STEMI). We used cardiovascular magnetic resonance $(\mathrm{CMR})$ to investigate this issue.

\section{Methods}

Fifteen STEMI patients undergoing successful primary percutaneous coronary intervention (pPCI) were included. Peripheral vein blood samples were drawn at 48 hours after pPCI to measure CPC (CD34+/ KDR +/CD45-). At 2-7 days after pPCI all patients underwent CMR assessment with SSFP, T2-STIR, first-pass perfusion and late gadolinium-enhancement imaging. Quantitative $\mathrm{T} 2$ area-at-risk (AAR, \% of LV volume), infarct size (IS, \% of LV volume), myocardial salvage (MS, calculated as AAR-IS) were calculated. Microvascular obstruction (MVO) was assessed both as perfusion defect score on first-pass imaging (PD, expressed as a sum of a 0 to 3 scoring for each of 16 LV segments) or as MVO score in the late enhancement images.

\section{Results}

CPC levels (expressed as \%, number of cells per total number of cytometric events) were more strongly related to extent of AAR ( $r h o=0.72, p=0.002$ ) than to IS (rho=0.5, p=0.06), but did not correlate to MS (rho $=0.002, \mathrm{p}=0.9)$. Moreover, $\mathrm{CPC}$ were related to PD score (rho=0.54, $\mathrm{p}=0.03$ ) but not to MVO score $(\mathrm{rho}=0.3, \mathrm{p}=0.2$ ).

\section{Conclusions}

Our data suggest that CPC are mobilized in STEMI patients in response to the total myocardial ischemic insult, as represented by the area-at-risk on T2-STIR. Furthermore, CPC positive correlation with PD suggests that they could be mobilized in attempt to overcome ongoing microvascular dysfunction.

Published: 2 February 2011

doi:10.1186/1532-429X-13-S1-P240

Cite this article as: Porto et al:: Peripheral levels of circulating progenitor cells correlate with area at risk and microvascular obstruction at cardiac magnetic resonance imaging in patients with STelevation myocardial infarction. Journal of Cardiovascular Magnetic Resonance 2011 13(Suppl 1):P240. 